\title{
Analysis of Rotman Lens Antenna for Different Substrates for Circular Contour
}

\author{
Shruti Vashist ${ }^{1}$ \\ ${ }^{1}$ Research Scholar, FET, ECE-Department, Manav Rachna International University, Faridabad, Haryana, India \\ e-mail:shruti.fet@mriu.edu.in \\ M.K.Soni ${ }^{2}$, P.K.Singhal ${ }^{3}$ \\ ${ }^{2}$ Executive Director \&Dean Manav Rachna International University, Faridabad, Haryana, India \\ e-mail:ed.fet@mriu.edu.in \\ ${ }^{3}$ HOD-ECE, MITS, Gwalior, India \\ pks_65@yahoo.com
}

\begin{abstract}
This paper presents a trifocal Rotman lens design approach. The effect due to change of substrate on the circular contour is observed. The shape of the beam contour is taken as circular. Different substrates can be used for the fabrication of the lens. Three different materials have been used to fabricate the lens antenna .A three beam prototype feeding five element antenna array working in ISM band has been simulated using RLD1.7.Effects on the performance of the antenna is observed.
\end{abstract}

Index Terms - Rotman lens antenna, RLD, Dielectric constant, substrate, array factor, phase error, side lobe level.

\section{INTRODUCTION}

Due to advancements in wireless communication technology there has been an increase in the demand of phased array systems for high performance Radar and Communication system. There has been a growing need for low cost and efficient phase shifting. Different active and passive beam forming networks (BFN's) are known from literature [1], [4] [25]. BFN's which are based on butler matrix are easy to construct and are also implementable on printed circuit boards, but the major drawback of these BFN's is that the produced beams are dependent on frequency and the beam shift occurs as frequency varies, which is not desirable in most communication links. Hardware complexity of these BFN's grows exponentially with the number of elements and hence it becomes difficult to realize such kind of BFN's for large number of radiation beams. To achieve low cost, reliable, multibeam phase arrays, the Rotman lens provides an effective solution [7][25]. Rotman lens is a true time delay beam former which provides linear phase shifts at the output ports by utilizing different paths within the lens structure. Rotman lens is a type of microwave lens and is capable of operating in high microwave frequency ranges with wide angle scanning capabilities. It is an Electronically Scanning Antenna (ESA) which has the ability to position the antenna beam instantaneously to any position and can also be implemented in the microstrip configuration. W.Rotman and R.Turner first proposed the Rotman lens which consisted of air filled parallel conducting plates fed by co-axial probes [1]. D. Archer [2] gave a modified design of Rotman lens in which a dielectric material is filled between parallel conducting plates fed by microstrip lines.

This paper presents the analysis of the performance of the Rotman lens due to change in the dielectric material which is filled between parallel conducting plates. Simulations were carried out using RLD1.7 designer software and various important parameters such as array factor, Side lobe level, insertion loss, S-parameter magnitude, beam to array port phase error, beam to array port coupling magnitude are analyzed to observe the performance of the lens. This paper is organized as follows: Section II presents the basic principle of the Rotman lens in which trifocal lens design equations and important parameters are discussed. Section III presents the effect of change in the dielectric material on the circular shaped contour. In Section IV the simulation and result analysis is done. Finally section $\mathrm{V}$ draws the conclusion.

\section{BASIC PRINCIPLE OF ROTMAN LENS}

\subsection{Lens Design Equation}

Fig. 1 shows a schematic diagram of a trifocal Rotman lens [14][25]. Input ports lie on contour $\mathrm{C}_{1}$ and the output ports lie on contour $\mathrm{C}_{2} . \mathrm{C}_{1}$ is known as beam contour and $\mathrm{C}_{2}$ is known as array contour. There are three focal points namely $F_{1}, F_{2}$ and $F_{3} . F_{1}$ is located on the central axis while $F_{2}$ and $F_{3}$ are symmetrically located on the beam contour at an angle of $+\alpha$ and $-\alpha$ respectively. ordinates of two off-axis focal points $\mathrm{F}_{2}, \mathrm{~F}_{3}$ and one on axis focal point $F_{1}$ are $\left(-f_{2} \cos \alpha, f_{2} \sin \alpha\right),\left(-f_{2} \cos \alpha,-f_{2} \sin \alpha\right)$ and $\left(-f_{1}, 0\right)$ respectively. 


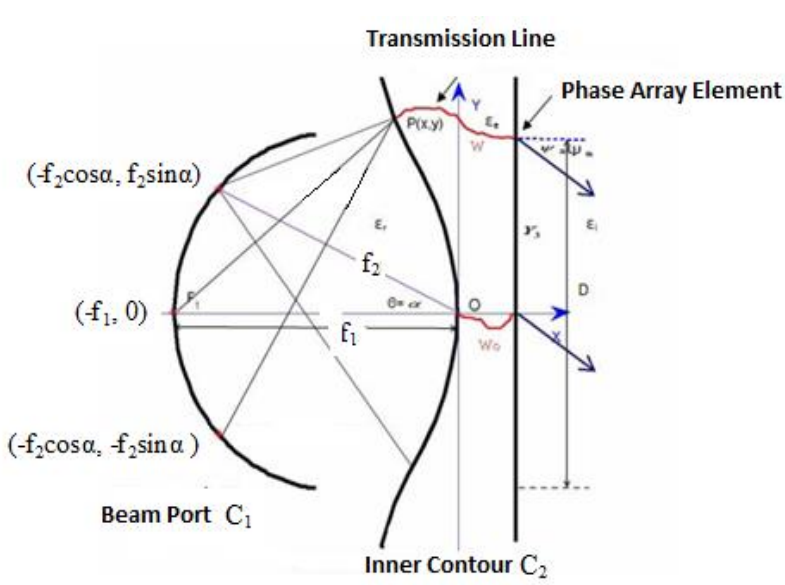

Fig.1 Schematic diagram of a trifocal Rotman lens

Where

$f_{1}$-On axis focal length

$f_{2}$-Off axis focal length

$\alpha$-Off center focal angle

$\psi_{\alpha}$-Scanning angle

$\gamma=\frac{\sin \psi}{\sin \alpha}$ - beam angle to ray angle ratio given as ratio of sine of their angles.

$\varepsilon_{r}$-Permittivity of medium in between the lens contour

$\varepsilon_{e}$ - Permittivity of medium of transmission line

$\varepsilon_{i}$-Permittivity of medium of radiating element

$\beta=\frac{\mathrm{f}_{2}}{\mathrm{f}_{1}}$-Focal ratio

$w_{o}$ - Transmission line length between axis point ' $\mathrm{O}$ ' and radiating element.

$w$-Transmission line length between points 'P'and radiating element.

$\mathrm{F}_{\mathrm{i}} \mathrm{P}-\mathrm{It}$ is the physical distance from focal point Fi to $\mathrm{P}$.

$\xi$ is an important parameter that relates the distance $\mathrm{Y}_{3}$ of any point on the array contour from the axis, to $f_{1}$. $\xi$ controls the portion of phase and amplitude error curves that the lens experiences [4].It is given by-

$$
\xi=\frac{Y_{3} \gamma}{f_{1}}
$$

If we assume that the ideal focal points are located at $\theta=$ $\pm \alpha$ and 0 , and their corresponding radiation angles are $\Psi= \pm \psi_{\alpha}$ and $\Psi=0$, given $\psi_{\alpha}$ is a known angle, simultaneous equations 1-3 are satisfied:

$$
F_{2} P \sqrt{\varepsilon_{r}}+w \sqrt{\varepsilon_{e}}+Y_{3} \sqrt{\varepsilon_{i}} \sin \psi_{\alpha}=f_{2} \sqrt{\varepsilon_{r}}+w_{o} \sqrt{\varepsilon_{e}} .
$$

$$
\begin{gathered}
F_{3} P \sqrt{\varepsilon_{r}}+w \sqrt{\varepsilon_{e}}-Y_{3} \sqrt{\varepsilon_{i}} \sin \psi_{\alpha}=f_{2} \sqrt{\varepsilon_{r}}+w_{o} \sqrt{\varepsilon_{e}} \\
F_{1} P \sqrt{\varepsilon_{r}}+w \sqrt{\varepsilon_{e}}=f_{1} \sqrt{\varepsilon_{r}}+w_{o} \sqrt{\varepsilon_{e}}
\end{gathered}
$$

Also from the above figure we have-

$$
\begin{array}{r}
\left(F_{3} P\right)^{2}=\left(-f_{2} \cos \alpha-X\right)^{2}+\left(Y+f_{2} \sin \alpha\right)^{2} \\
\left(F_{2} P\right)^{2}=\left(-f_{2} \cos \alpha-X\right)^{2}+\left(Y-f_{2} \sin \alpha\right)^{2} \\
\left(F_{1} P\right)^{2}=\left(-f_{1}-X\right)^{2}+Y^{2}
\end{array}
$$

By algebraic manipulation of the above equations the geometric lens equation which is quadratic in nature is given as [6][7][8][25]

$$
a \frac{\varepsilon_{r}}{\varepsilon_{e}} w^{2}+b \frac{\sqrt{\varepsilon_{r}}}{\sqrt{\varepsilon_{e}}} w+c=0
$$

Hence the length of transmission line is calculated as

$$
w=\frac{\sqrt{\varepsilon_{e}}}{\sqrt{\varepsilon_{r}}} \frac{\left(-b \pm \sqrt{b^{2}-4 a c}\right)}{2 a}
$$

And the values of ' $x$ ' and ' $y$ ' are calculated as

$$
\begin{array}{r}
x=\frac{(1-\beta) \mathrm{w} \sqrt{\varepsilon_{e}}}{(\beta \cos \alpha-1) \sqrt{\varepsilon_{r}}}+\frac{y_{3}^{2} \varepsilon_{i} \sin ^{2} \Psi_{\alpha}}{2(\beta \cos \alpha-1) \varepsilon_{r}} \\
y=\frac{\xi \sqrt{\varepsilon_{i}}}{\sqrt{\varepsilon_{r}}}\left(1-\frac{w \sqrt{\varepsilon_{e}}}{\beta \sqrt{\varepsilon_{r}}}\right) \\
a=1-\frac{(1-\beta)^{2}}{(1-\beta C)^{2}}-\frac{\xi^{2} \varepsilon_{i}}{\beta^{2} \varepsilon_{r}} \\
b=-2+\frac{2 \xi^{2} \varepsilon_{i}}{\beta \varepsilon_{r}}-\frac{(1-\beta) \xi^{2} S^{2} \varepsilon_{i}}{(1-\beta C)^{2} \varepsilon_{r}}+\frac{2(1-\beta)}{(1-\beta C)} \\
\mathrm{c}=\left[\frac{y_{3}^{4} \sin ^{4} \psi_{\alpha} \varepsilon_{i}^{2}}{4(\beta C-1)^{2} \varepsilon_{r}{ }^{2}}+\frac{\xi^{2} \varepsilon_{i}}{\varepsilon_{r}}+\frac{y_{3}{ }^{2} \sin ^{2} \psi_{\alpha} \varepsilon_{i}}{(\beta C-1) \varepsilon_{r}}\right]
\end{array}
$$

When a feed is placed at a non-focal point then the corresponding wavefront will have a phase error, but for wide angle scanning capabilities it is necessary to place 
the feed at non focal points [10]. The number of beams, number of elements, maximum beam angle and element spacing are known from the system requirement. Element spacing $\mathrm{d}$ is also very critical as it controls the appeariance of grating lobes [5]. The spacing that just admits a grating lobe is given by-

$$
\frac{d}{\lambda} \text { 战 } \frac{1}{2+\sin \Psi_{m}}
$$

Where $\Psi_{\mathrm{m}}$ is the maximum beam angle.

\section{EFFECT OF CHANGE OF SUBSTRATE ON THE CIRCULAR SHAPED CONTOUR}

The dielectric constant of the lens substrate supports both the parallel plate region and the transmission lines. The upper limit of the dielectric constant is set at 50 as this is the limit of validity for the equations in the software. Dielectric constant of the substrate plays an important role in the overall performance of the antenna. The more desirable substrates are generally thick substrates with low dielectric constant [9][20] .They show better efficiency, bandwidth and performance of the antenna, but then the size of the antenna increases. Thin substrates with higher dielectric constant are desirable to minimize undesired radiation and coupling and will lead to small element size. A tradeoff has to be achieved so as to have good performance and circuit design. Loss tangent or dissipation factor also affects the performance of the antenna. It is used in calculation of port coupling and Array factor. Rotman Lens is designed using RLD s/w to operate in the frequency band of $2300 \mathrm{MHz}-2500$ $\mathrm{MHz}$ with the centre frequency of $2.4 \mathrm{GHz}$. The lens is analysed to find the effect of change in the substrate keeping the element spacing and focal ratio constant. The height of array contour and feed contour must be almost same in order to couple maximum power from the feed contour to the array contour [5]. Taking this fact into consideration the alpha ratio of the designed lens is kept at 1.0 .

The specifications of the designed lens are as follows:-

Element spacing $=0.0625 \mathrm{mts}$

Operating frequency $=2.4 \mathrm{GHz}$

Scan angle $=30$ degree

Alpha ratio $=1.0$

No. of beam ports $=3$

No. of array ports $=5$

Flare angle $=12$ degree

Focal length $=0.2031 \mathrm{mts}$

Focal ratio $(\mathrm{g})=1.1$

Beam port Excitation-Port 2

In order to analyse the effect of change of the substrate on the performance of the Rotman Lens, three different materials are used with different dielectric constant and loss tangent. The shape of the lens contour is taken as circular[15] .The change of the substrate will affect the
Rotman lens parameters like Side Lobe Level, phase error, insertion loss and S-parameter magnitude. The analysis of all the lens parameters is done.

i) RT Duroid: RT Duroid is Glass Microfiber Reinforced PTFE (poly tetra fluoro ethylene) composite produced by Roger Corporation. RT Duroid 5870 substrate has low loss tangent. They exhibit excellent chemical resistance, including solvent and reagents used in printing and plating, ease of fabrication cutting, shearing, machining, and environment friendly[16]. The permittivity of the material is 2.2 and $\tan \delta=0.0004$

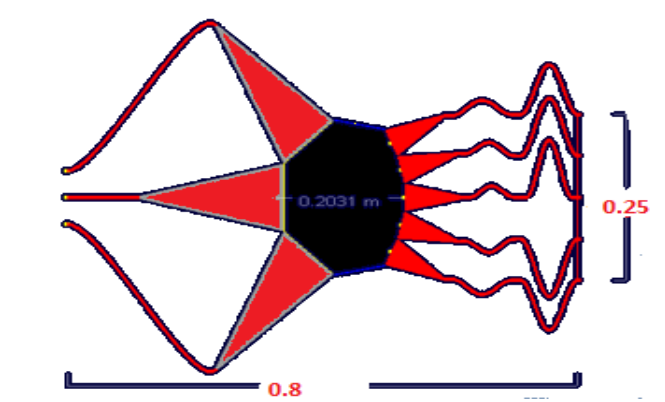

Fig. 2 Circular contour with permittivity of the material 2.2 and $\tan \delta=0.0004$

a. Array Factor: Array factor is an important factor for the analysis of Rotman Lens performance. Array factor analysis indicates the behaviour of side lobe levels and the scanning directions [19]. The side lobe level of a circular contour is $-14 \mathrm{db}$ in case of RT Duroid for all the frequencies under consideration. Figure 3 shows the array factor plot for element spacing of $0.0625 \mathrm{~m}$. When the element spacing is increased, the number of side lobes and their level both increase which is undesirable. The grating lobe starts to appear which have almost the same before \& after, for authors names font size is 11 , affiliations font size is 10 level as the main lobe.

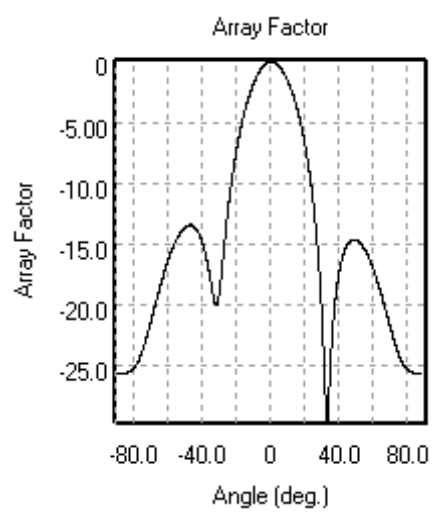

Fig. 3Array factor

b. Beam to array phase error: Fig.4 Shows beam to array port phase error when Port 2 is excited. Table 1.1 summarizes the beam to array port phase error for different substrates. Maximum phase error is observed for port 4 and port 8 and minimum phase error is for port 6 . Alpha ratio is kept 1.0 so that the height of the array and the beam contour remains almost same, hence maximum power is coupled from beam to array port. 


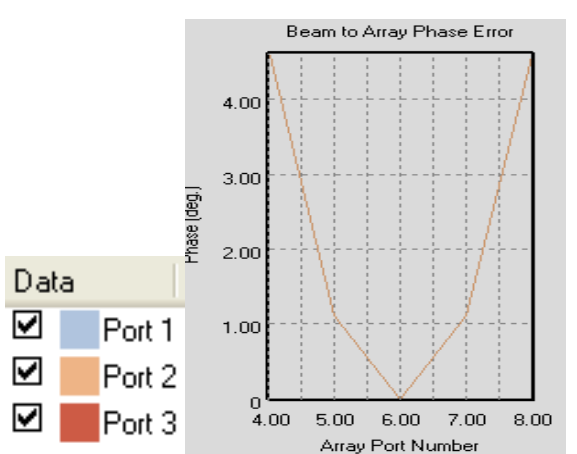

Fig 4.Beam to array phase error

c. Beam to array coupling amplitude: Fig.5 Shows beam to array coupling magnitude for port 2 excitation .Maximum coupling is observed for port 6.Table 1.2 shows the beam to array coupling magnitude for port 2 excitation for different substrates.

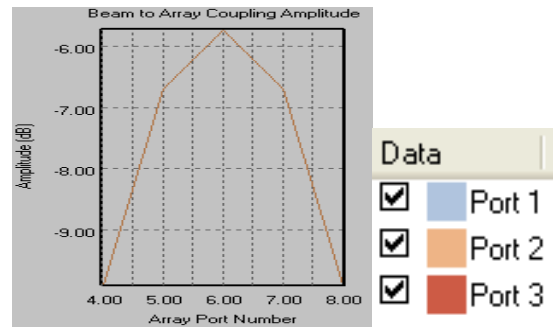

Fig 5 Beam to array Coupling amplitude

d. Insertion loss: The insertion loss is calculated by summing the received powers at the array and beam ports, relative to the transmitted power of each beam [26]. The insertion loss is calculated by-

$$
\mathrm{L}_{\mathrm{k}}=-10 \log \sum_{\mathrm{n}}\left|\mathrm{S}_{\mathrm{nk}}\right|^{2}
$$

This is the insertion loss corresponding to beam port $\mathrm{k}$ where $n$ is the index for the array ports [26]. Fig 6 shows the insertion loss variation for beam port 2 in the operating frequency band .Its value is $1.865 \mathrm{db}$ at $2.4 \mathrm{GHz}$.

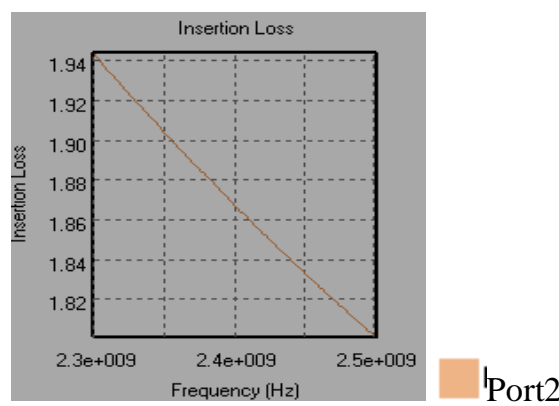

Fig. 6 Insertion loss $(1.865 \mathrm{db}$ at $2.4 \mathrm{GHz})$

e. S-Parameter: $S_{22}$ parameter represents the return loss for the system. It indicates the ratio of actual power that has been transmitted through the system to amount of the power that is reflected back to the system [17]. In matrix form the magnitude of S-parameters can be represented as a $3 * 8$ matrix

$$
\left[\begin{array}{llllllll}
S_{11} & S_{12} & S_{13} & S_{14} & S_{15} & S_{16} & S_{17} & S_{18} \\
S_{21} & S_{22} & S_{23} & S_{24} & S_{25} & S_{26} & S_{27} & S_{28} \\
S_{31} & S_{32} & S_{33} & S_{34} & S_{35} & S_{36} & S_{37} & S_{38}
\end{array}\right]
$$

In this case $\mathrm{S}_{22}$ magnitude represents the return loss for port 2. Its value is found to be $-12.342 \mathrm{db}$. Fig.7 shows the plot of magnitude of $S_{22}$ with frequency.

$$
\left[\begin{array}{cccccccc}
-12.34 & 0 & 0 & -7.69 & -7.84 & -9.98 & -14.58 & -26.81 \\
0 & -12.34 & 0 & -10.78 & -7.57 & -6.62 & -7.57 & -10.70 \\
0 & 0 & -12.34 & -26.89 & -14.58 & -9.98 & -7.84 & -7.61
\end{array}\right]
$$

The value of $\mathrm{S}_{22}$ calculated as $-12.34 \mathrm{~dB}$ which shows that only $5.8 \%$ of the incident power is reflected back to port 2 and maximum power is transmitted which is $94.2 \%$. Fig.7 shows the plot of magnitude of $S_{22}$ with frequency.

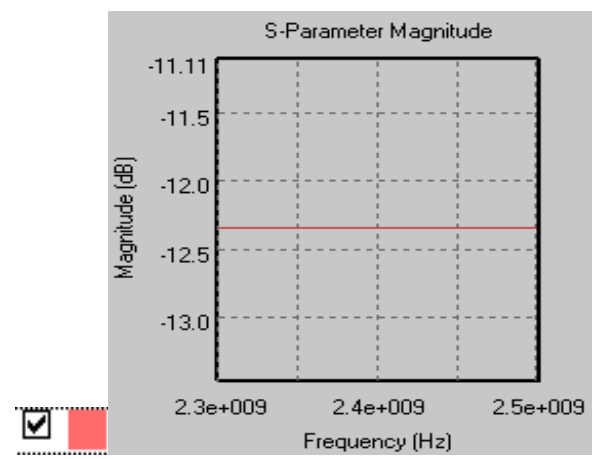

Fig 7 Magnitude at S22

ii) FR4-Gloss Epoxy: FR4 is a grade designation assigned to glass -reinforced epoxy laminate sheets, tubes, rods and printed circuit boards (PCB). FR 4 is a composite material composed of woven fibreglass cloth with an epoxy resin binder that is flame resistant (selfextinguishing).

The value of permittivity is 4.4 and $\tan \delta=0.013$.

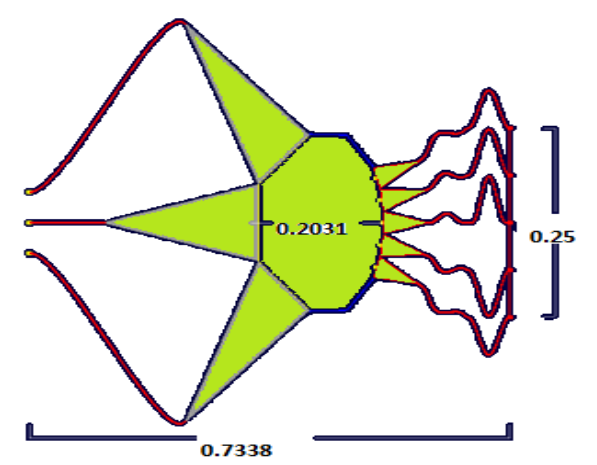

Fig. 8 Circular contour with permittivity is 4.4 and $\tan \delta=0.013$

a. Array factor: Fig.9 shows the improvement in the side lobe level which has come upto $-14.8 \mathrm{db}$.So it can be observed that as the value of permittivity increases that side lobe level improves for the circular contour. 


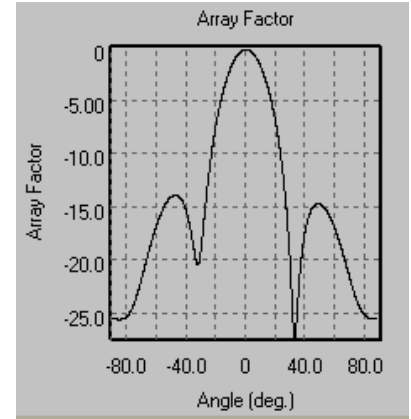

Fig.9 Array Factor

b. Beam to array phase error: Beam to array plot of FR4 is shown in fig 10 and the analysis is given in Table 1.1 .

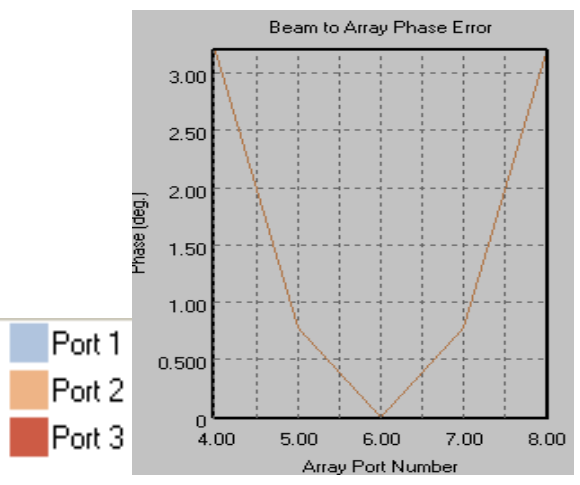

Fig.10 Beam to array phase error

c. Beam to array coupling: Beam to array coupling is shown in fig 11 and the analysis is given in Table1.2.

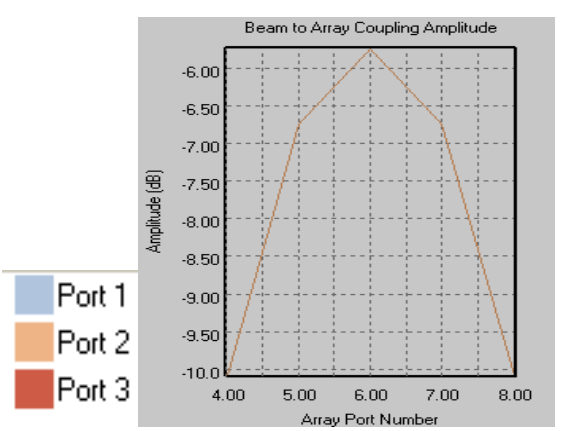

Fig.11 Beam to array Coupling amplitude

d. Insertion loss: Insertion loss for FR4 is $4.077 \mathrm{db}$ as shown in fig. 12.

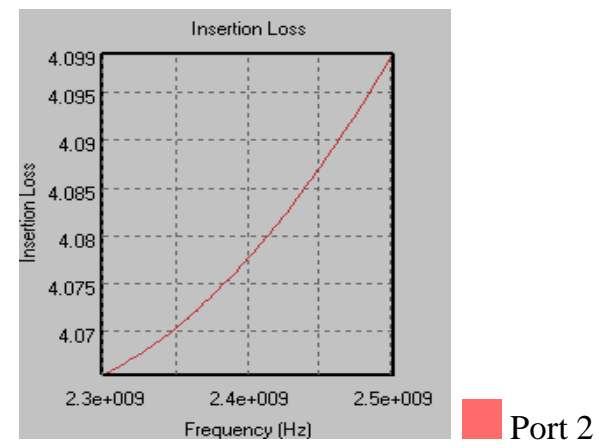

Fig.12 Insertion loss $(4.077 \mathrm{db}$ at $2.4 \mathrm{GHz})$ e. S-Parameter:The S-parameter or return loss for FR4 is calculated from plot given in fig 13.

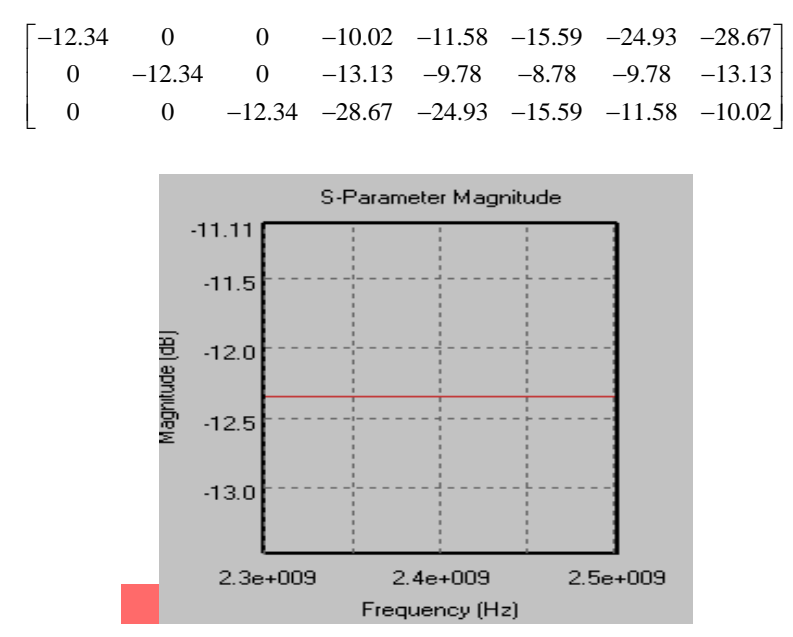

Fig.13 S-parameter-Magnitude (-12.34db )

iii) Bakelite: It is a thermosetting phenol formaldehyde resin, formed from an elimination reaction of phenol with formaldehyde. It is most commonly used as an electrical insulator possessing considerable mechanical strength[23]. The value of permittivity is 4.78 and $\tan \delta=0.03045$.

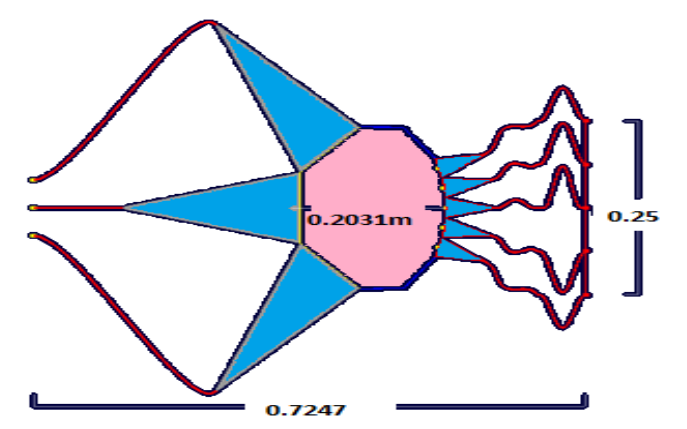

Fig 14 Circular contour with permittivity 4.78 and $\tan \delta=0.03045$

a. Array factor:The plot for Bakelite is shown in fig 14.The side lobe level is at $-14.9 \mathrm{db}$ which is better than the substrate with permittivity 4.4 .

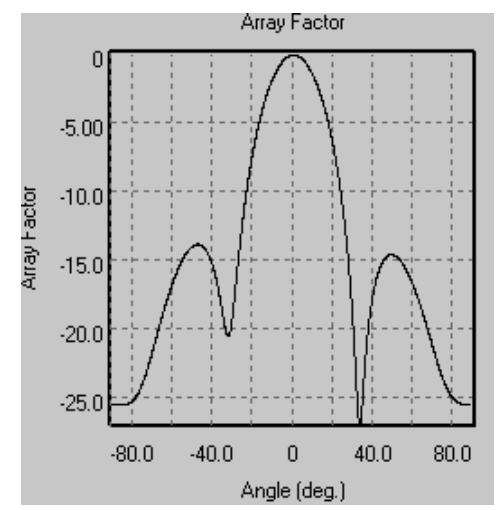

Fig.14 Array Factor

b. Beam to array phase error: Fig 15 shows beam to array phase error and analysis is shown in table 1.1 . 


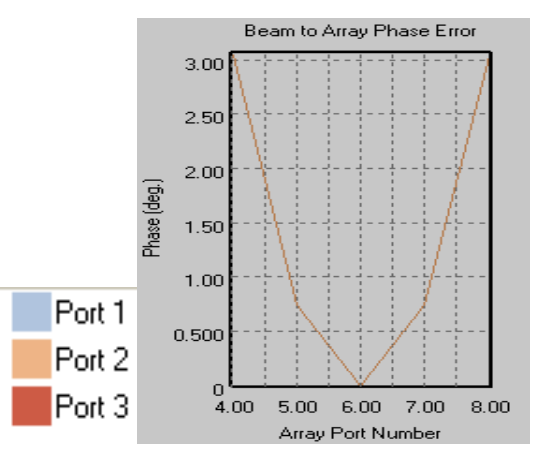

Fig.15 Beam to array phase error

c. Beam to array coupling magnitude: Beam to array coupling magnitude is given in fig 16 and analysis is done in table 1.2.

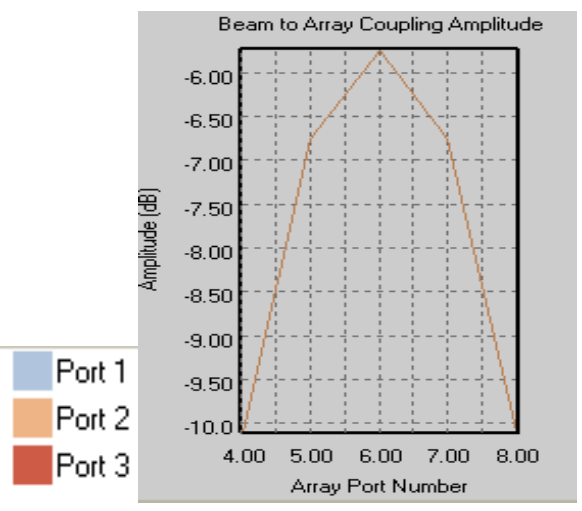

Fig.16 Beam to array Coupling magnitude

d. Insertion loss: Plot for insertion loss is shown in fig 17.Insertion loss for Bakelite is 7.08.

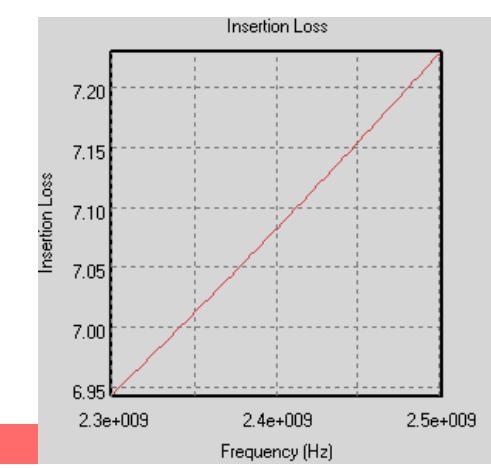

Fig.17 Insertion loss ( $7.08 \mathrm{db}$ at $2.4 \mathrm{GHz})$

e. S-Parameter: S-Parameter is shown in fig 18.Analysis of S-parameter is shown in the matrix below.

$$
\left[\begin{array}{cccccccc}
-12.34 & 0 & 0 & -13.25 & -14.95 & -19.29 & -30.21 & -29.37 \\
0 & -12.34 & 0 & -16.15 & -12.78 & -11.78 & -12.78 & -16.15 \\
0 & 0 & -12.34 & -29.37 & -30.21 & -19.29 & -14.95 & -13.25
\end{array}\right]
$$

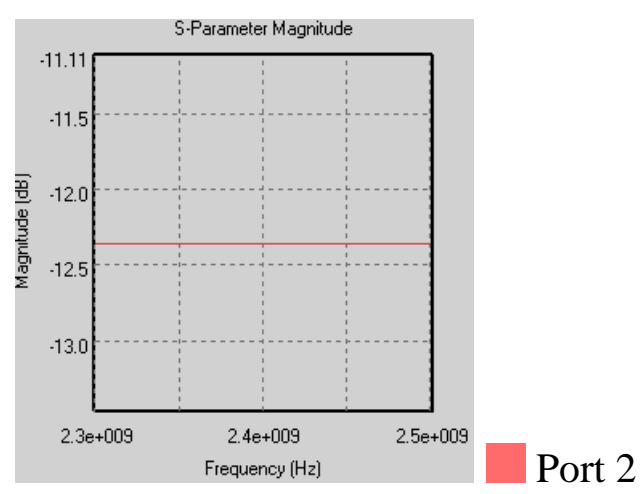

Fig.18 S-parameter Magnitude

\section{SIMULATION RESULT: THE ANALYSIS OF VARIOUS PARAMETERS ARE TABULATED BELOW.}

Table 1.1 Analysis of Phase error for circular contour

\begin{tabular}{|c|c|c|c|c|}
\hline \multirow{4}{*}{$\begin{array}{l}\mathrm{I} / \mathrm{P} \\
\text { Port }\end{array}$} & \multirow{4}{*}{$\begin{array}{l}\mathrm{O} / \mathrm{P} \\
\text { Port }\end{array}$} & $\begin{array}{l}\text { Phase } \\
\text { error in } \\
\text { (deg) }\end{array}$ & $\begin{array}{l}\text { Phase } \\
\text { error in } \\
\text { (deg) }\end{array}$ & $\begin{array}{l}\text { Phase } \\
\text { error in } \\
(\mathrm{deg})\end{array}$ \\
\hline & & $\begin{array}{c}\text { RT } \\
\text { Duroid }\end{array}$ & FR-4 & Bakelite \\
\hline & & $\varepsilon_{r}=2.2$ & $\varepsilon_{r}=4.4$ & $\varepsilon_{r}=4.78$ \\
\hline & & $\begin{aligned} & \tan \delta \\
= & 0.0004\end{aligned}$ & $\begin{array}{c}\tan \delta \\
=0.013\end{array}$ & $\begin{array}{c}\tan \delta \\
=0.03045\end{array}$ \\
\hline 2 & 4 & 4.5 & 3.2 & 3.1 \\
\hline 2 & 5 & 1.2 & 0.75 & 0.7 \\
\hline 2 & 6 & 0 & 0 & 0 \\
\hline 2 & 7 & 1.2 & 0.75 & 0.7 \\
\hline 2 & 8 & 4.5 & 3.2 & 3.1 \\
\hline
\end{tabular}

It can be observed from table 1.1 that for a given circular contour, as the permittivity increases the phase error decreases. Hence the value of permittivity should be chosen such that the overall phase error reduces.

Table 1.2 Beam to array coupling magnitude for circular contour

\begin{tabular}{|c|c|c|c|c|}
\hline \multirow{3}{*}{$\begin{array}{l}\text { I/P } \\
\text { Port }\end{array}$} & \multirow{3}{*}{$\begin{array}{l}\text { O/P } \\
\text { Port }\end{array}$} & $\begin{array}{l}\text { Coupling } \\
\text { magnitude } \\
(\mathrm{db})\end{array}$ & $\begin{array}{l}\text { Coupling } \\
\text { magnitude } \\
(d b)\end{array}$ & $\begin{array}{l}\text { Coupling } \\
\text { magnitude } \\
(d b)\end{array}$ \\
\hline & & $\varepsilon_{r}=2.2$ & $\varepsilon_{r}=4.4$ & $\mathcal{E}_{r}=4.78$ \\
\hline & & $\begin{array}{l}\tan \boldsymbol{\delta} \\
=0.0004\end{array}$ & $\tan \delta=0.013$ & $\begin{array}{l}\tan \boldsymbol{\delta} \\
=0.03045\end{array}$ \\
\hline 2 & 4 & -10 & -10.1 & -10.1 \\
\hline 2 & 5 & -6.61 & -6.62 & -6.79 \\
\hline 2 & 6 & -5.7273 & -5.74 & -5.7406 \\
\hline 2 & 7 & -6.61 & -6.62 & -6.79 \\
\hline 2 & 8 & -10 & -10.1 & -10.1 \\
\hline
\end{tabular}


In case of circular contour as the value of permittivity increases the coupling magnitude increases. The coupling magnitude with reference to port 4 and port 8 initially remains same for all values of permittivity later it increases .In rest of the cases i.e. for port 5 to port 8 the coupling magnitude increases with the increase in the permittivity.

Table 1.3 SLL and insertion loss

\begin{tabular}{|l|l|l|}
\hline \multirow{2}{*}{ Substrate } & $\begin{array}{l}\text { Insertion } \\
\text { loss(db) }\end{array}$ & SLL(db) \\
\cline { 2 - 3 } & Circular lens & $\begin{array}{l}\text { Circular } \\
\text { lens }\end{array}$ \\
\hline$\epsilon_{\mathrm{r}=2.2}$ & 1.865 & -14 \\
\hline$\epsilon_{\mathrm{r}=3.2}$ & 2.165 & -14.37 \\
\hline$\epsilon_{\mathrm{r}=3.4}$ & 2.18 & -14.75 \\
\hline$\epsilon_{\mathrm{r}=4.4}$ & 4.077 & -14.8 \\
\hline$\epsilon_{\mathrm{r}=4.78}$ & 7.08 & -14.9 \\
\hline
\end{tabular}

The insertion loss increases as the permittivity increases. The side lobe level is better in case of the circular contour. There is a rise in the side lobes if the permittivity increases.

\section{CONCLUSION}

A design approach for trifocal Rotman Lens has been presented in this paper making use of different substrate like Bakelite, FR4 and RT Duroid with different value of permittivity .The shape of the lens is kept circular .Rotman Lens prototype with 3 beam ports and 5 array ports has been simulated using RLD1.7 designer software. A simulation result shows the effect of change in the substrate. Various important parameters like array factor, side lobe level, phase error, coupling magnitude, insertion loss and S-parameter have been analysed. It has been clearly seen that for the circular contour the array factor plot showing the side lobe level improves as the value of the permittivity increases for various substrate. The formation of grating lobe is not there .The phase error plot shows that as the value of the permittivity increases the phase error decreases sharply. The insertion loss increases as the permittivity increases. The coupling magnitude also shows some kind of sharp variation. Hence a trade-off has to be kept between the permittivity and the insertion loss. Analysis of $S$ parameters reveals that the return loss of the designed Rotman Lens is $-12.34 \mathrm{~dB}$ for port $2\left(\mathrm{~S}_{22}\right)$. This indicates that only $5.8 \%$ of the incident power is reflected back to port 2 and $94.2 \%$ of the power is successfully transmitted through the system.

\section{REFERENCES}

[1] W. Rotman and R. F. Turner, "Wide angle microwave lens for line source application", IEEE Trans. 1963, pp. 623-630.
[2] D.H.Archer,'Lens-fed multiple beam arrays",Microwave J,.PP.171- 95,Sept1984.

[3] J.Kim and F.S.Barns"Dielectric slab Rotman lens with tapered slot antenna array",IEEE prop-Microwave Antenna Prop.vol.152,no.6,PP.557-562,Dec 2005.

[4] R.C.Hansen,'Design trades for Rotman lenses",IEEE Trans Antennas prop vol.39,no.4,April 1991,PP.464-472.

[5] P. K. Singhal, P.C. Sharma, and R. D. Gupta, "Rotman lens with equal height of array and feed contours", IEEE Transaction on Antennas and Propagation, vol. 51, Issue 8, pp. 2048-2056, Aug. 2003.

[6] P. C. Sharma et al., "Two-dimensional field analysis for CAD of Rotman-type beam-forming lenses," Int. J. Microw. Millim.-Wave CAD Eng., vol. 2, no. 2, pp. 9097, 1992.

[7] E. O. Rausch, A. F. Peterson, W. Wiebach: Electronically Scanned Millimeter Wave Antenna Using A Rotman Lens. Radar 97, pp 374-379, October 1997, Publication No. 449.

[8] Fuchs H.-H.; Nüßler D..Design of Rotman lens for BeamSteering of $94 \mathrm{GHz}$ Antenna Array, Electronic Letters 27th May 1999 Vol. 35 No.11.

[9] L. Musa and M. S. Smith, "Microstrip port design and sidewall absorption for printed Rotman lens", Microwaves, Antennas and Propagation, IEEE Proceeding, vol. 136, Issue 1, pp. 53-58, Feb. 1989.

[10] T. Katagi, S. Mano and S.I. Sato, "An Improved Design Method of Rotman Lens Antennas", IEEE Transactions on Antennas and Propagation, 1984, vol. ap-32,no. 5, pp. 524-527.

[11] D. Nußler, H.H. Fuchs, and R. Brauns, "Rotman lens for the millimetre wave frequency range," in Proc. Eur. Microw. Conf., Oct. 2007, pp. 696-699.

[12] J. Dong, A. I. Zaghloul, and R. Rotman, "Non-Focal Minimum-Phase- Error Planar Rotman Lens," in URSI National Radio Science Meeting Colorado, 2008.

[13] A. I. Zaghloul and J. Dong, "A Concept for a Lens Configuration for 360-Degree Scanning," IEEE Letters on Antennas and Wireless Propagation, 2009.

[14] J. Dong and A. I. Zaghloul, "Implementation of Microwave Lens for 360-Degree Scanning," in IEEE International Symposium on Antennas Propagation Charleston, South Carolina, 2009.

[15] R.C. Hansen, "Phased Array Antennas", New York: John Wiley and Sons, 1998.

[16] S.S.D. Jones, H. Gent and A.A.L. Browne, "Improvements in or Relating to Electromagnetic-Wave Lens and Mirror Systems", British Provisional Patent Specification No. 25926/56, August 1956.

[17] G.L. Leonakis, "Correction to Wide-Angle Microwave Lens for Line Source Applications", IEEE Transactions on Antennas and Propagation, vol. 36, no. 8, p.1067, August 1986.

[18] K.K. Chan, "A Feed Network for Multibeam Triangular Grid and Hexagonal Shape Planar Array Antennas", IEEE Transactions on Antennas and Propagation, 1999, pp. 8083, vol.1.

[19] L. Hall, H. Hansen and D. Abbott, "Rotman Lens for mmWavelengths", Proc. SPIE, 2002, vol. 4935, pp. 215-221.

[20] J. Dong and A.I. Zahghloul, "Extremely High-Frequency Beam Steerable Lens-Fed Antenna for Vehicular Sensor Applications", IET Microwaves, Antennas \&Propagation, 2009.

[21] D.R. Gagnon, "Procedure for Correct Refocusing of the Rotman Lens According to Snell's Law", IEEE Transactions on Antennas and Propagation, 1989,vol. 37, 
no. 3, pp. 390-392.

[22] J. Kim and F. S. Barnes, "Scaling and Focusing of the Rotman Lens", Antennas and Propagation Society International Symposium, IEEE, 2001, vol. 2, pp. 773-776.

[23] Rogers Corporation Advanced Circuit Materials Division, Data Sheet RO1.4000, Revised 2002, Publication \#92-004, "RO4000R Series High Frequency Circuit Materials", "http://www.apcircuits.com/resources/information/ro4000 ds 4.pdf".

[24] E.O. Rausch and A.F. Peterson, "Rotman Lens Design Issues", Antennas and Propagation Society International Symposium, IEEE, 2005, vol. 2B, pp. 35-38.

[25] Shruti Vashist,Umesh Dutta,M.K.Soni,'Design and performance analysis of Rotman Lens,Internatioanl Journal of Engineering research and applications, Vol2 issue 4,1792-1795.

[26] Shruti Vashist, M.K.Soni, P.K.Singhal'Rotman Lens performance analysis,ACEEE Internatioanl Journal of Signal and Image Processing,Vol5 issue 1,Jan 2014.

\section{Authors' Profiles}

Shruti Vashist, Associate prof at the Faculty of Engineering and technology at Manav Rachna International University. She is currently working towards the Ph.D. degree in the Rotman lens antenna at the Manav Rachna international University. Her research interests include Wireless and Mobile Communication, mobile computing.

Dr. M.K. Soni did h is B.Sc (Engg.) in 1972 and M.Sc (Engg.) in 1975 from REC Kurukshetra (Now NIT Kurukshetra) and thereafter completed his Ph.D from REC Kurukshetra (in collaboration with IIT Delhi) in 1988. He has a total 39 years of rich experience into Academics. His area of interest is microprocessor based control systems and digital system design. He has more than 150 research papers in the International and National Journals to his credit. Presently he is Executive Director \& Dean, Faculty of Engineering and Technology, Manav Rachna International University, Faridabad.

Prof. P.K. Singhal received his B.E. and Ph.D. Degrees in Electronics Engineering from Jiwaji University, Gwalior (MP)India, in 1987 and 1997, respectively, and the M.Tech degree in Microwave Electronics from the University of Delhi, India in 1989. Currently, he is presently Head of the department of Electronics Engineering at Madhav Institute of Technology and Science, Gwalior(MP)-India. He has about more than 100 publications to his credit at the national and International level. His research interest includes electromagnetic, antennas, microwave circuits \& communication systems.

How to cite this paper: Shruti Vashist, M.K.Soni, P.K.Singhal,"Analysis of Rotman Lens Antenna for Different Substrates for Circular Contour", IJIGSP, vol.6, no.8, pp. 53-60, 2014.DOI: 10.5815/ijigsp.2014.08.08 